\title{
Jet spectroscopy of buckybowl: Electronic and vibrational structures in the S0 and S1 states of triphenylene and sumanene
}

\section{$\operatorname{AUTHOR(S):~}$}

Kunishige, Sachi; Kawabata, Megumi; Baba, Masaaki; Yamanaka, Takaya; Morita, Yuki; Higashibayashi, Shuhei; Sakurai, Hidehiro

\section{CITATION:}

Kunishige, Sachi ... [et al]. Jet spectroscopy of buckybowl: Electronic and vibrational structures in the S0 and S1 states of triphenylene and sumanene. The Journal of chemical physics 2013, 139(4): 044313.

\section{ISSUE DATE:}

2013-07-28

URL:

http://hdl.handle.net/2433/178529

\section{RIGHT:}

(C) 2013 American Institute of Physics. 


\section{AIP $\begin{gathered}\text { mesoumalor } \\ \text { chemical Physics }\end{gathered}$}

Jet spectroscopy of buckybowl: Electronic and vibrational structures in the S0 and S1 states of triphenylene and sumanene

Sachi Kunishige, Megumi Kawabata, Masaaki Baba, Takaya Yamanaka, Yuki Morita et al.

Citation: J. Chem. Phys. 139, 044313 (2013); doi: 10.1063/1.4816636

View online: http://dx.doi.org/10.1063/1.4816636

View Table of Contents: http://jcp.aip.org/resource/1/JCPSA6/v139/i4

Published by the AIP Publishing LLC.

Additional information on J. Chem. Phys.

Journal Homepage: http://jcp.aip.org/

Journal Information: http://jcp.aip.org/about/about_the_journal

Top downloads: http://jcp.aip.org/features/most_downloaded

Information for Authors: http://jcp.aip.org/authors

\section{ADVERTISEMENT}

\section{AIP | Applied Physics Letters}

Accepting Submissions in

Biophysics and Bio-Inspired Systems 


\title{
Jet spectroscopy of buckybowl: Electronic and vibrational structures in the $S_{0}$ and $S_{1}$ states of triphenylene and sumanene
}

\author{
Sachi Kunishige, ${ }^{1}$ Megumi Kawabata, ${ }^{1}$ Masaaki Baba, ${ }^{1, a)}$ Takaya Yamanaka, ${ }^{2}$ \\ Yuki Morita, ${ }^{2}$ Shuhei Higashibayashi, ${ }^{2}$ and Hidehiro Sakurai ${ }^{2}$ \\ ${ }^{1}$ Division of Chemistry, Graduate School of Science, Kyoto University, Kyoto 606-8502, Japan \\ ${ }^{2}$ Institute for Molecular Science, Okazaki 444-8585, Japan
}

(Received 26 March 2013; accepted 11 July 2013; published online 31 July 2013)

\begin{abstract}
Sumanene is a typical buckybowl molecule with $C_{3 v}$ symmetry. We observed a fluorescence excitation spectrum and a dispersed fluorescence spectrum of sumanene in a supersonic jet. Bowl effects were clarified by comparing the spectra with those of triphenylene $\left(D_{3 h}\right.$ symmetry), which is a planar prototype of nonplanar sumanene. The $S_{1}{ }^{1} A_{1} \leftarrow S_{0}{ }^{1} A_{1}$ transition is symmetry allowed. We found the $0_{0}^{0}$ band in the fluorescence excitation spectrum at $357.78 \mathrm{~nm}$; this band was missing in the forbidden $S_{1}{ }^{1} A_{1}^{\prime} \leftarrow S_{0}{ }^{1} A_{1}^{\prime}$ transition of triphenylene. The transition moment was shown to be along the oblate symmetric top axis (out of plane) by the observed rotational contour. A large number of vibronic bands were observed, unlike in triphenylene. Some were considered to be out-of-plane vibrational modes, which lead to a bowl-to-bowl inversion reaction assisted by in-plane vibrations. We found that the vibronic bands were markedly weak in the high energy region of triphenylene- $d_{12}$. This indicates that the fluorescence quantum yield is very low at the high vibrational levels in the $S_{1}$ state due to the rapid radiationless transition. The main process is considered to be internal conversion to the $S_{0}$ state. The nonplanar structural distortion may also enhance radiationless transitions. We could not, however, observe weakening of the vibronic bands in the fluorescence excitation spectrum of sumanene. (C) 2013 AIP Publishing LLC. [http://dx.doi.org/10.1063/1.4816636]
\end{abstract}

\section{INTRODUCTION}

Buckybowls are newly synthesized and unique polycyclic aromatic hydrocarbons (PAHs) that possess bowlshaped round $\pi$ systems. ${ }^{1-5}$ They are of great interest to both chemical physicists and organic chemists because of their characteristic nonplanar structure and unique dynamical processes, such as the bowl-to-bowl inversion reaction. ${ }^{6-10}$ Prototypical molecules are sumanene and corannulene, which are especially important as fragment models of buckyball fullerene. ${ }^{11,12}$ We are interested in the bowl effect, which is defined as the specific change in chemical and physical properties with its out-of-plane distortion of a $\pi$ frame. Here, we focus on sumanene, whose molecular structure is illustrated in Fig. 1(a). This molecule is nonplanar and it has a three-fold symmetry axis ( $C_{3 v}$ point group).

A $\pi$-conjugated planar molecule with a three-fold symmetry ( $D_{3 h}$ point group) has its own peculiar character in the electronic molecular orbitals and vibrational modes. It is impossible to distinguish their components along the two in-plane coordinate axes $(x, y)$. The HOMO and LUMO are represented by $E^{\prime \prime}$, which is a second-order irreducible representation, and the actual electronic structure is complicated. Only the ${ }^{1} E^{\prime} \leftarrow{ }^{1} A_{1}^{\prime}$ absorption is an allowed $\pi \pi^{\star}$ transition in the ground state of a neutral $D_{3 h}$ molecule. Even for the forbidden transition, many vibronic bands are expected to be observed due to vibronic interaction with other electronic

\footnotetext{
a) Author to whom correspondence should be addressed. Electronic mail: baba@kuchem.kyoto-u.ac.jp
}

excited states. Nonradiative processes can be induced by coupling between the excited and ground states. If the $\pi$ frame is distorted out of plane, nonradiative processes are expected to be further enhanced by the mixing of in-plane and out-ofplane molecular orbitals and vibrational modes. As such, it is very important to investigate the electronic and vibrational structures of the isolated molecule. We observed the fluorescence excitation spectrum and the dispersed fluorescence spectrum of sumanene in a supersonic jet.

A planar prototype of sumanene is triphenylene, the molecular structure of which is illustrated in Fig. 1(b). We performed similar spectroscopic studies of triphenylene- $h_{12}$ and triphenylene- $d_{12}$ in supersonic jets. The electronic and vibrational structures have been clarified in several reports on the spectroscopic studies of triphenylene in cold materials ${ }^{13,14}$ and in a supersonic jet. ${ }^{15}$ Theoretical calculations predict that the $S_{1}$ state is ${ }^{1} A_{1}^{\prime}$ and the $S_{1}{ }^{1} A_{1}^{\prime} \leftarrow S_{0}{ }^{1} A_{1}^{\prime}$ electronic $\pi \pi^{\star}$ transition is symmetry forbidden. It is, therefore, necessary to consider the vibronic interaction between the $S_{1}$ and another excited state in terms of the appearance of numerous vibronic bands. Sumanene is nonplanar and the $S_{1}{ }^{1} A_{1} \leftarrow S_{0}{ }^{1} A_{1}$ electronic transition is allowed with the transition moment along the oblate symmetric top axis $(c)$. The nonradiative processes are also expected to differ from those of a planar $\pi$-rich molecule.

In this article, we present the experimental results of the jet spectroscopy of triphenylene and sumanene and analyses on the basis of theoretical calculations. We discuss the bowl effect of a buckybowl molecule, sumanene, by comparing the electronic and vibrational structures with triphenylene. 
(a) Sumanene

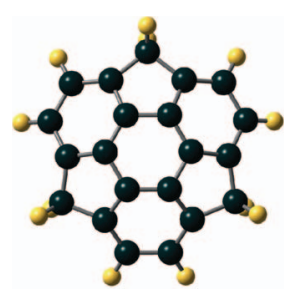

(b) Triphenylene
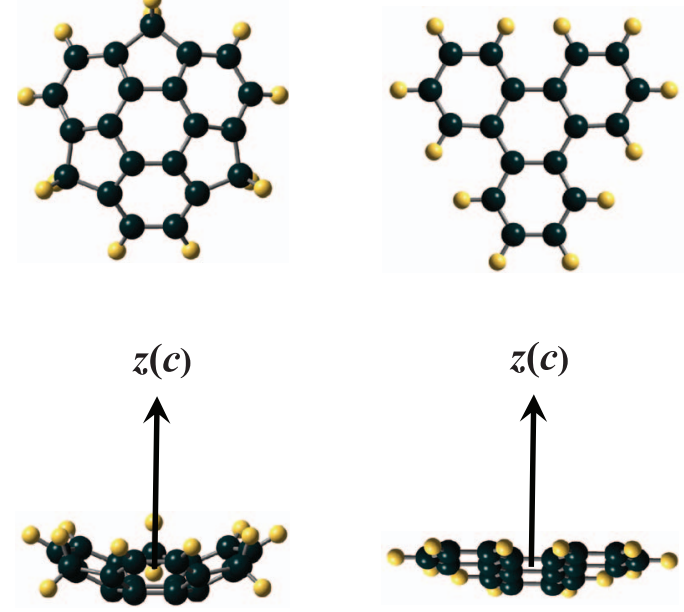

FIG. 1. Molecular structures and top axes $(z(c))$ of (a) sumanene and (b) triphenylene.

\section{EXPERIMENTAL}

Sumanene was synthesized by following the method described elsewhere, which is based on oxidative aromatization using tetrahedral $\mathrm{sp}^{3}$ carbons starting from norbornadiene, ${ }^{11}$ and recrystallized from dichloromethane. Commercially obtained triphenylene- $h_{12}$ (Aldrich, 98\%) and triphenylene- $d_{12}$ (CDN ISOTOPES, 98.4\%) were used without further purification. The solid sample was heated in a stainless steel container (approximately $150^{\circ} \mathrm{C}$ ) and the vapour was mixed with $\mathrm{He}$ gas $(99.9 \%, 2 \mathrm{~atm})$. The mixed gas was expanded into a high-vacuum chamber through a pulsed nozzle (an automobile electric fuel injector) to generate a supersonic jet. The jet was crossed with a pulsed laser light beam at right angles. The distance between the nozzle and the crossing point was $1 \mathrm{~cm}$. Fluorescence from excited molecules was focused onto a photomultiplier surface (Hamamatsu R928) using a pair of lenses through a glass color filter to block scattered laser light.

The current output of the photomultiplier was amplified using a preamplifier (Stanford Research SR240). The electric signal of fluorescence intensity was obtained using a boxcar integrator (Stanford Research SR250), and the change with the laser light wavelength was recorded as a fluorescence excitation spectrum. A dispersed fluorescence spectrum was observed using a scanning monochromator (Nikon P250) with tuning the laser light wavelength to a specific vibronic band.

A tunable dye laser (Lambda Physik, LPD3000, $\Delta E=0.1 \mathrm{~cm}^{-1}$, Exalite 348 ) was employed as a light source that was pumped by a pulsed excimer laser (Coherent, Compex Pro $110, \Delta t=5 \mathrm{~ns}, 308 \mathrm{~nm}, 200 \mathrm{~mJ}$ ) for $340-380 \mathrm{~nm}$, and a tunable dye laser with an opto-parametric amplifier (Lambda Physik, Scanmate-OPPO, $\Delta E=0.1 \mathrm{~cm}^{-1}$ ) and an SHG crystal (potassium dihydrogen phosphate (KDP)) for $315-340 \mathrm{~nm}$. The wavelength of laser light was calibrated by a mercury lamp and a monochromator. The accuracy of absolute wavelength of an observed band is $\pm 1 \mathrm{~cm}^{-1}$.

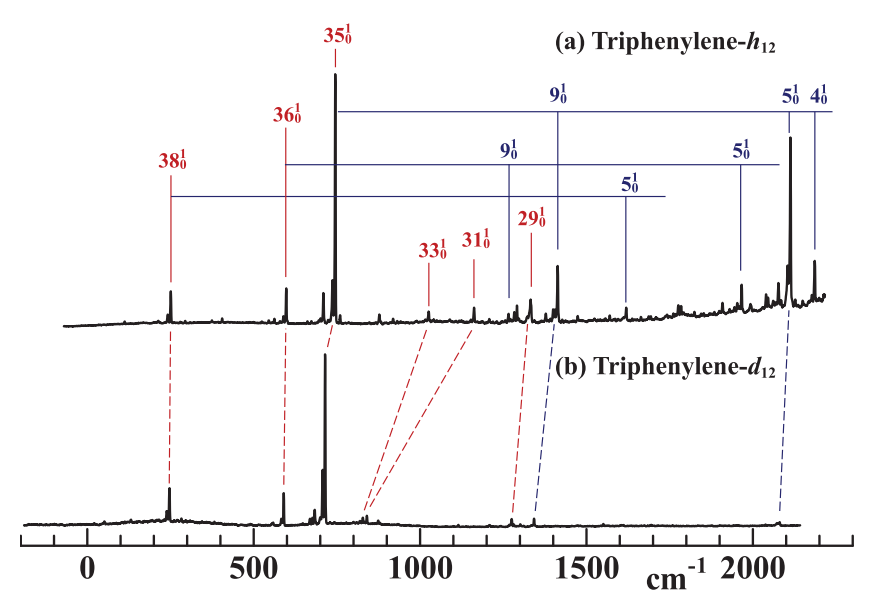

FIG. 2. Fluorescence excitation spectra of the $S_{1}{ }^{1} A_{1}^{\prime} \leftarrow S_{0}{ }^{1} A_{1}^{\prime}$ transitions of jet-cooled (a) triphenylene- $h_{12}$ and (b) triphenylene- $d_{12}$.

\section{RESULTS AND ANALYSIS}

\section{A. Triphenylene- $h_{12}$ and Triphenylene- $d_{12}$}

The $S_{1} \leftarrow S_{0}$ transition of triphenylene can be observed in the wavelength region of 315-340 nm. We observed fluorescence excitation spectra of triphenylene- $h_{12}$ and triphenylene$d_{12}$ in supersonic jets; the results are shown in Fig. 2. In order to analyze the observed spectral feature, it is necessary to identify the $S_{1}$ electronic state. First, we performed theoretical calculations of excited states using the GAUSSIAN 09 program package. ${ }^{16}$ It was shown that the molecule was planar and three-fold symmetric by several trial calculations with geometry optimization. We employed the results of the timedependent density functional theory (TDDFT)(B3LYP)/6$31 \mathrm{G}$ calculation, retaining $D_{3 h}$ symmetry. Figure 3(a) depicts the calculated $\pi$ molecular orbitals. The results of the excited states, excitation wavelengths, oscillator strengths, and coefficients of main configurations are listed in Table I. The direct product of $\operatorname{HOMO}\left(E^{\prime \prime}\right)$ and $\operatorname{LUMO}\left(E^{\prime \prime}\right)$ irreducible representations can be reduced as

$$
E^{\prime \prime} \otimes E^{\prime \prime}=A_{1}^{\prime}+A_{2}^{\prime}+E^{\prime} .
$$

The $S_{1}$ state is expected to be ${ }^{1} A_{1}^{\prime}\left(\pi \pi^{\star}\right)$, which is mainly represented by $\Phi_{59}\left(\right.$ HOMO: $\left.E^{\prime \prime}\right) \rightarrow \Phi_{61}\left(\right.$ LUMO: $\left.E^{\prime \prime}\right)$ and $\Phi_{60}\left(\right.$ HOMO: $\left.E^{\prime \prime}\right) \rightarrow \Phi_{62}\left(\right.$ LUMO: $\left.E^{\prime \prime}\right)$ excitations. The $0_{0}^{0}$ band is missing in the spectrum because the ${ }^{1} A_{1}^{\prime} \rightarrow{ }^{1} A_{1}^{\prime}$ transition is forbidden in $D_{3 h}$ symmetry. An allowed $\pi \pi^{\star}$ transition from the $S_{0}$ state is only ${ }^{1} E^{\prime} \leftarrow{ }^{1} A_{1}^{\prime}$, whose transition moment is along the molecular plane. The $e^{\prime}$ vibrational levels in the $S_{1}{ }^{1} A_{1}^{\prime}$ state can be observed by mixing with the $S_{5}$ or $S_{6}{ }^{1} E^{\prime}$ state. ${ }^{17,18}$ Consequently, the observed prominent vibronic bands are assigned to $e^{\prime}$ and the combination bands with the totally symmetric $a_{1}^{\prime}$ vibration based on an analogy of benzene. The vibronic assignments are listed in Table II.

One difficulty is that it is impossible to experimentally determine the absolute wavenumber of the $0_{0}^{0}$ band for a forbidden transition. We, therefore, made good use of theoretical calculations of vibrational energies. The $e^{\prime}$ vibronic bands can first be assigned unambiguously by referring to the results of TDDFT(B3LYP)/6-31G with geometry optimization. Their observed wavenumbers were least-squares fit for both 


\section{(a) Triphenylene}
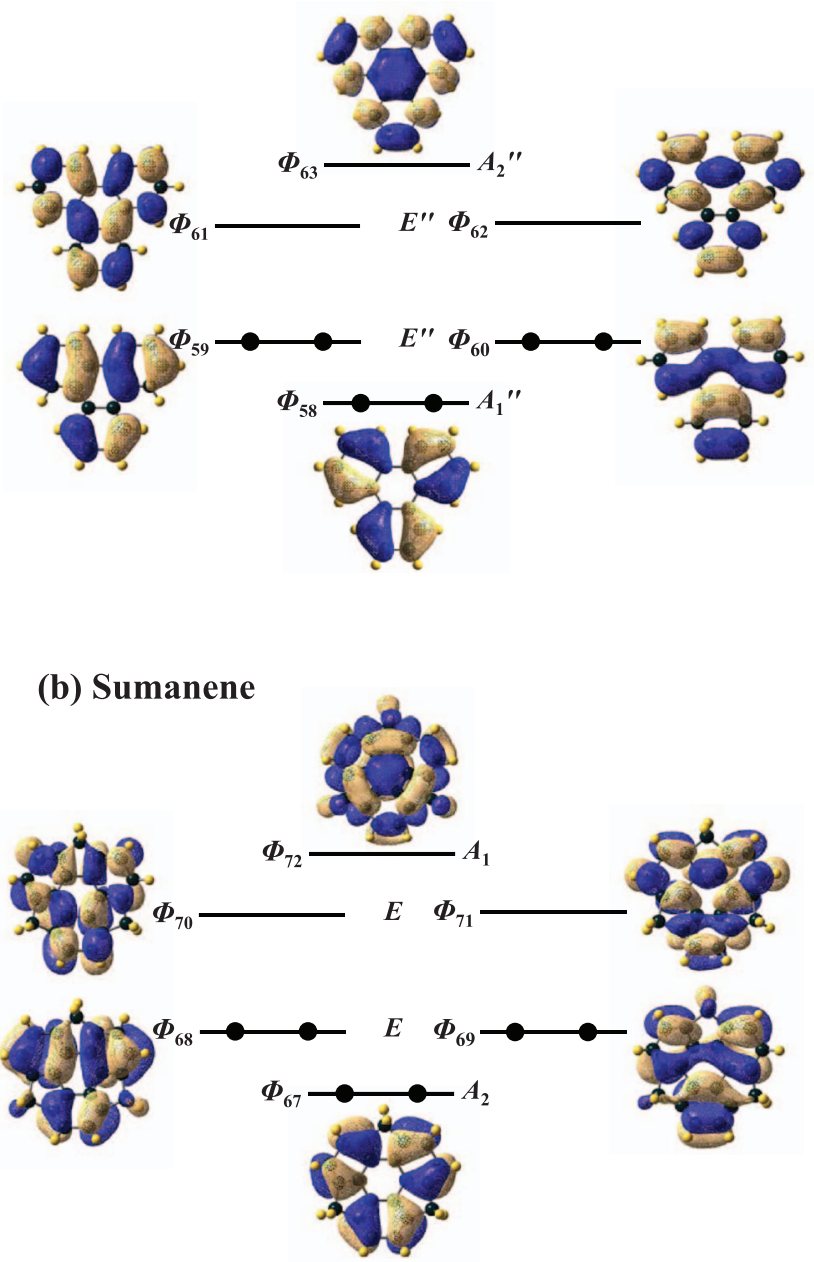

FIG. 3. $\pi$ molecular orbitals of (a) triphenylene and (b) sumanene.

triphenylene- $h_{12}$ and triphenylene- $d_{12}$. The best results were obtained by using a scaling factor of 0.961 for the calculated vibrational energies. Consequently, we could estimate the absolute wavenumbers of the $0_{0}^{0}$ bands, respectively, to be 29618 and $29695 \mathrm{~cm}^{-1}$. The former value is identical to the

TABLE I. Excited states, excitation wavelengths, oscillator strengths, and coefficients of the main configurations of triphenylene calculated by TDDFT(B3LYP)/6-31G.

\begin{tabular}{lcrc}
\hline \hline Excited state & Excitation wavelength & Oscillator strength \\
\hline$S_{1}{ }^{1} A_{1}^{\prime}$ & $326.53 \mathrm{~nm}$ & & $f=0.0000$ \\
& $\Phi_{59} \rightarrow \Phi_{61}$ & -0.4951 & \\
& $\Phi_{60} \rightarrow \Phi_{62}$ & 0.4951 & \\
$S_{2}{ }^{1} A_{2}^{\prime}$ & $306.26 \mathrm{~nm}$ & & $f=0.0000$ \\
& $\Phi_{59} \rightarrow \Phi_{62}$ & 0.4950 & \\
& $\Phi_{60} \rightarrow \Phi_{61}$ & 0.4961 & \\
$S_{3}{ }^{1} A_{1}^{\prime}$ & $282.09 \mathrm{~nm}$ & & $f=0.0000$ \\
& $\Phi_{58} \rightarrow \Phi_{61}$ & -0.3625 & \\
$S_{4}{ }^{1} A_{2}^{\prime}$ & $\Phi_{60} \rightarrow \Phi_{63}$ & -0.5928 & \\
& $282.04 \mathrm{~nm}$ & & $f=0.0000$ \\
& $\Phi_{58} \rightarrow \Phi_{62}$ & -0.3624 & \\
$S_{5}{ }^{1} E^{\prime}$ & $\Phi_{59} \rightarrow \Phi_{63}$ & 0.5926 & \\
$S_{6}{ }^{1} E^{\prime}$ & $263.84 \mathrm{~nm}$ & & $f=0.4640$ \\
\hline \hline
\end{tabular}

TABLE II. Observed and calculated vibrational energies $\left(\mathrm{cm}^{-1}\right)$ and assignments in the $S_{1}{ }^{1} A_{1}^{\prime}$ states of triphenylene- $h_{12}$ and triphenylene- $d_{12}$.

\begin{tabular}{lcl}
\hline \hline $\begin{array}{l}\text { Triphenylene- } h_{12} \\
\text { Obs }^{\mathrm{a}}\left(\mathrm{Calc}^{\mathrm{b}}\right)\end{array}$ & $\begin{array}{c}\text { Triphenylene- } d_{12} \\
\text { Obs }^{\mathrm{a}}\left(\mathrm{Calc}^{\mathrm{b}}\right)\end{array}$ & \multicolumn{1}{c}{ Assignment } \\
\hline 0 & 0 & $\begin{array}{c}0_{0}^{0}\left(h_{12}: 29618 \mathrm{~cm}^{-1},\right. \\
\left.d_{12}: 29695 \mathrm{~cm}^{-1}\right)^{\mathrm{c}}\end{array}$ \\
$253(251)$ & $242(235)$ & $38_{0}^{1}\left(e^{\prime}\right)$ \\
$598(606)$ & $585(584)$ & $36_{0}^{1}\left(e^{\prime}\right)$ \\
$709(716)$ & $678(677)$ & $35_{2}^{1}$ \\
$745(745)$ & $709(700)$ & $35_{0}^{1}\left(e^{\prime}\right)$ \\
$1026(1027)$ & $824(832)$ & $33_{0}^{1}\left(e^{\prime}\right)$ \\
$1162(1156)$ & $835(841)$ & $31_{0}^{1}\left(e^{\prime}\right)$ \\
$1266(1276)$ & & $90_{0}^{1} 36_{0}^{1}$ \\
$1332(1320)$ & $1270(1250)$ & $290_{0}^{1} /\left(e^{\prime}\right)$ \\
$1405(1400)$ & & $27_{0}^{1} /\left(e^{\prime}\right)$ \\
$1413(1415)$ & $1337(1333)$ & $9_{0}^{1} 35_{0}^{1}$ \\
$1619(1598)$ & & $5_{0}^{1} 38_{0}^{1}$ \\
$1784(1785)$ & & $8_{0}^{1} 35_{0}^{1}$ \\
$1930(1922)$ & & $7_{0}^{1} 35_{0}^{1}$ \\
$1966(1953)$ & & $5_{0}^{1} 36_{0}^{1}$ \\
$2113(2092)$ & & $5_{0}^{1} 35_{0}^{1}$ \\
$2186(2197)$ & & $4_{0}^{1} 35_{0}^{1}$ \\
\hline \hline
\end{tabular}

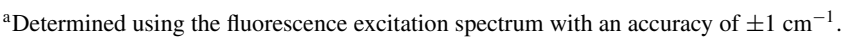
${ }^{\mathrm{b}}$ Results of TDFFT(B3LYP)/6-31G are scaled by 0.961 .

${ }^{\mathrm{c}}$ Estimated by a least-squares fit of the observed vibrational energies to the calculated ones. See text.

value given by Kokkin et al., ${ }^{15}$ and this estimation is considered to be reliable. The blue shift of $77 \mathrm{~cm}^{-1}$ by full deuterium substitution seems to be reasonable for this size of molecule. ${ }^{19}$ We assigned other vibronic bands as well, and the results are listed in Table II together with the calculated vibrational energies. We use the same mode numbers as those used by Kokkin et al. ${ }^{15}$

The prominent bands in the low energy region are assigned to the $e^{\prime}$ fundamental bands of $\nu_{35}, v_{36}$, and $\nu_{38}$. Their vibrational energies are $5 \%$ reduced by full deuteration, whereas the energies of $v_{31}$ and $v_{33}$ are considerably reduced (approximately 20\%); their vibronic bands are observed at around $1000 \mathrm{~cm}^{-1}$. We assigned the 1405 and $1413 \mathrm{~cm}^{-1}$ bands to $27_{0}^{1}\left(e^{\prime}\right)$ and $9{ }_{0}^{1} 35_{0}^{1}$, respectively, although these assignments are different from those by Kokkin et al. ${ }^{15}$ They assigned the stronger $1413 \mathrm{~cm}^{-1}$ band to $27_{0}^{1}$ because the $v_{9}$ progression could not be identified. A number of strong bands are assigned to the combination bands of $a_{1}^{\prime}$ and $e^{\prime}$. Here, we could assign the $9_{0}^{1} 36_{0}^{1}$ and the $9{ }_{0}^{1} 35_{0}^{1}$ band is considered to be stronger than the $27_{0}^{1}$ band. We assigned the $2113 \mathrm{~cm}^{-1}$ band to $5_{0}^{1} 35_{0}^{1}$ because the calculated vibrational energy of $v_{4}$ by TDDFT/6-31G is $84 \mathrm{~cm}^{-1}$ larger than the observed value of this band. Although the calculated $v_{5}$ energy is $30 \mathrm{~cm}^{-1}$ smaller, this assignment is considered to be more favorable. The $2186 \mathrm{~cm}^{-1}$ band is consequently assigned to $4{ }_{0}^{1} 35_{0}^{1}$.

In order to confirm this analysis and determine the vibrational energies in the $S_{0}$ state, we observed dispersed fluorescence spectra for the $35_{0}^{1}$ band excitation. The observed spectra were depicted in Fig. 4. We performed vibronic 


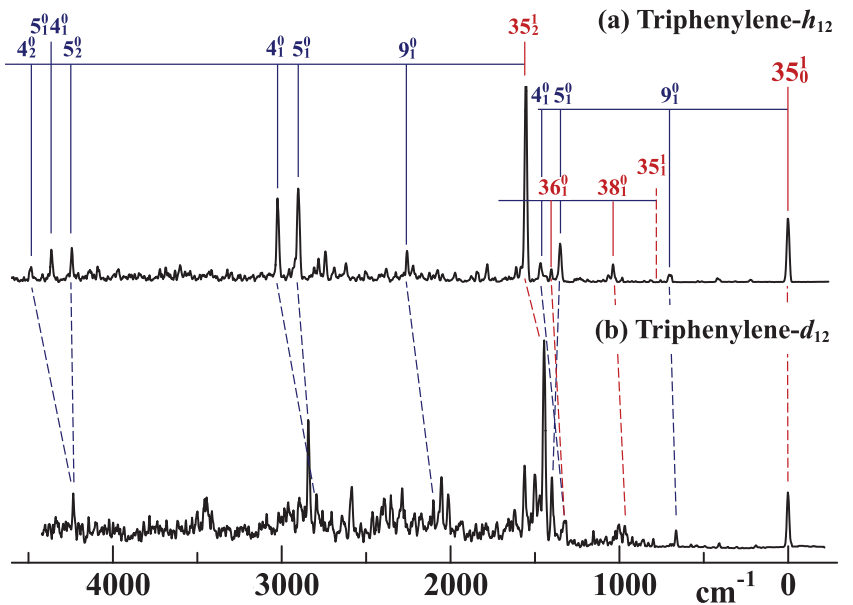

FIG. 4. Dispersed fluorescence spectra of jet-cooled (a) triphenylene- $h_{12}$ and (b) triphenylene- $d_{12}$ for the $35_{0}^{1}$ band excitation.

analysis by referring to the calculated values of vibrational energies in the $S_{0}{ }^{1} A_{1}^{\prime}$ state by (DFT)(B3LYP)/6-31G with geometry optimization. Here, we used a scaling factor of 0.968 , which was determined by a least-squares fit of the observed vibrational energies for both triphenylene- $h_{12}$ and triphenylene- $d_{12}$. The observed vibronic bands and the resultant assignments are listed in Table III. We could assign the $a_{1}^{\prime}$ fundamental bands of $v_{4}, v_{5}, v_{6}, \nu_{8}$, and $\nu_{9}$ as well as the

TABLE III. Observed and calculated vibrational energies $\left(\mathrm{cm}^{-1}\right)$ and assignments in the $S_{0}{ }^{1} A_{1}^{\prime}$ states of triphenylene- $h_{12}$ and triphenylene- $d_{12}$.

\begin{tabular}{lcl}
\hline \hline $\begin{array}{l}\text { Triphenylene- } h_{12} \\
\text { Obs }^{\mathrm{a}}\left(\text { Calc }^{\mathrm{b}}\right)\end{array}$ & $\begin{array}{c}\text { Triphenylene- } d_{12} \\
\text { Obs }^{\mathrm{a}}\left(\mathrm{Calc}^{\mathrm{b}}\right)\end{array}$ & \multicolumn{1}{c}{ Assignment } \\
\hline 0 & 0 & $35_{0}^{1}\left(h_{12}: 30363 \mathrm{~cm}^{-1}\right.$ \\
$\left.d_{12}: 30405 \mathrm{~cm}^{-1}\right)$ \\
$705(702)$ & $665(661)$ & $9_{1}^{0} 35_{0}^{1}\left(a_{1}^{\prime}\right)^{\mathrm{c}}$ \\
$1036(1039)$ & $967(972)$ & $35_{1}^{1} 38_{1}^{0}\left(e^{\prime}\right)^{\mathrm{d}}$ \\
$1350(1343)$ & $1399(1374)$ & $5_{1}^{0} 35_{0}^{1}\left(a_{1}^{\prime}\right)$ \\
$1400(1412)$ & $1325(1338)$ & $35_{1}^{1} 36_{1}^{0}\left(e^{\prime}\right)$ \\
$1466(1458)$ & $1317(1327)$ & $4_{1}^{0} 35_{0}^{1}\left(a_{1}^{\prime}\right)$ \\
$1552(1558)$ & $1446(1458)$ & $35_{2}^{1}$ \\
$1842(1842)$ & $1503(1502)$ & $31_{1}^{0} 35_{0}^{1}\left(a_{1}^{\prime}\right)$ \\
$2075(2088)$ & $1562(1579)$ & $35_{1}^{1} 33_{1}^{0}\left(e^{\prime}\right)$ \\
$2257(2260)$ & $2015(2024)$ & $35_{1}^{1} 29_{1}^{0}\left(e^{\prime}\right)$ \\
$2618(2630)$ & $2102(2119)$ & $35_{2}^{1} 9_{1}^{0}$ \\
$2688(2686)$ & $2354(2366)$ & $35_{2}^{1} 8_{1}^{0}$ \\
2740 & & $5_{2}^{0} 35_{0}^{1}$ \\
$2781(2796)$ & & \\
$2902(2901)$ & & $35_{2}^{1} 6_{1}^{0}$ \\
$3024(3016)$ & $2584(2612)$ & $35_{2}^{1} 5_{1}^{0}$ \\
$4243(4244)$ & $2843(2832)$ & $35_{2}^{1} 4_{1}^{0}$ \\
$4364(4359)$ & $2794(2785)$ & $35_{2}^{1} 5_{2}^{0}$ \\
$4484(4474)$ & $4234(4206)$ & $35_{2}^{1} 5_{1}^{0} 4_{1}^{0}$ \\
\hline \hline
\end{tabular}

${ }^{a}$ Determined using the dispersed fluorescence spectrum with an accuracy of $\pm 6 \mathrm{~cm}^{-1}$.

${ }^{\mathrm{b}}$ Results of DFT(B3LYP)/6-31G are scaled by 0.968 .

${ }^{\mathrm{c}}\left(a_{1}^{\prime}\right)$ means the $a_{1}^{\prime}$ fundamental band.

d $\left(e^{\prime}\right)$ means the $e^{\prime}$ fundamental band.

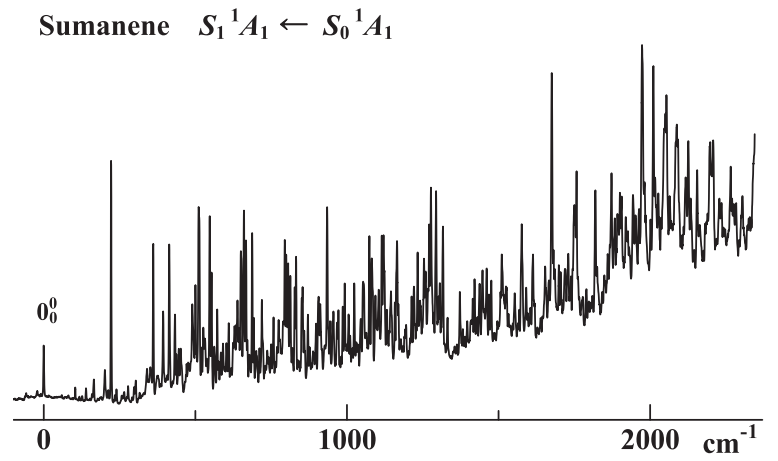

FIG. 5. Fluorescence excitation spectrum of the $S_{1}{ }^{1} A_{1} \leftarrow S_{0}{ }^{1} A_{1}$ transition of jet-cooled sumanene.

$e^{\prime}$ bands of $v_{33}, v_{35}, v_{36}$, and $v_{38}$. As the laser wavelength was tuned to the $35_{0}^{1}$ band, the $35_{2}^{1}$ and its combination bands with $a_{1}^{\prime}$ vibrations of $v_{4}, v_{5}, v_{6}$, and $v_{9}$ were strongly observed. The observed energies are in good agreement with the calculated values for both triphenylene- $h_{12}$ and triphenylene- $d_{12}$. The $35_{1}^{1}$ band is forbidden and missing in the dispersed fluorescence spectrum.

\section{B. Sumanene}

Figure 5 depicts the fluorescence excitation spectrum of sumanene in a supersonic jet, whose feature is clearly different from that of triphenylene. First, we found the $0_{0}^{0}$ band of the $S_{1} \leftarrow S_{0}$ transition at $357.78 \mathrm{~nm}$, although the intensity was relatively weak. Second, the spectrum was congested with a large number of vibronic bands in the all vibrational energy region. In the same manner as triphenylene, we performed theoretical calculations of excited states with geometry optimization at the TDDFT(B3LYP)/6-31G level in order to identify the $S_{1}$ state. The calculated $\pi$ molecular orbitals are illustrated in Fig. 3(b). The results of calculation for excited states, excitation wavelengths, oscillator strengths, and coefficients of main configurations are listed in Table IV. It is expected that the $S_{1}$ state is the ${ }^{1} A_{1}\left(\pi \pi^{\star}\right)$ state and is

TABLE IV. Excited states, excitation wavelength, oscillator strength, and coefficients of main configurations of sumanene calculated by TDDFT(B3LYP)/6-31G.

\begin{tabular}{lcrc}
\hline \hline Excited state & Excitation wavelength & & Oscillator strength \\
\hline$S_{1}{ }^{1} A_{1}$ & $336.62 \mathrm{~nm}$ & & $f=0.0004$ \\
& $\Phi_{68} \rightarrow \Phi_{70}$ & 0.4890 & \\
& $\Phi_{69} \rightarrow \Phi_{70}$ & 0.4885 & \\
$S_{2}{ }^{1} A_{2}$ & $317.25 \mathrm{~nm}$ & & $f=0.0000$ \\
& $\Phi_{68} \rightarrow \Phi_{71}$ & -0.4898 & \\
& $\Phi_{69} \rightarrow \Phi_{70}$ & 0.4906 & \\
$S_{3}{ }^{1} A_{1}$ & $302.84 \mathrm{~nm}$ & & $f=0.0002$ \\
& $\Phi_{67} \rightarrow \Phi_{70}$ & -0.2213 & \\
& $\Phi_{69} \rightarrow \Phi_{72}$ & 0.6618 & \\
$S_{4}{ }^{1} A_{2}$ & $282.04 \mathrm{~nm}$ & & $f=0.0000$ \\
& $\Phi_{67} \rightarrow \Phi_{71}$ & -0.3624 & \\
$S_{5}{ }^{1} E$ & $\Phi_{68} \rightarrow \Phi_{72}$ & -0.6618 & \\
$S_{6}{ }^{1} E$ & $279.04 \mathrm{~nm}$ & & $f=0.3784$ \\
\hline \hline
\end{tabular}


(a) $0_{0}^{0}$ Band

(b) $61_{0}^{1}(e)$ Band

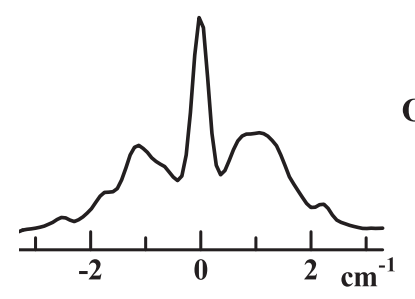

Obs
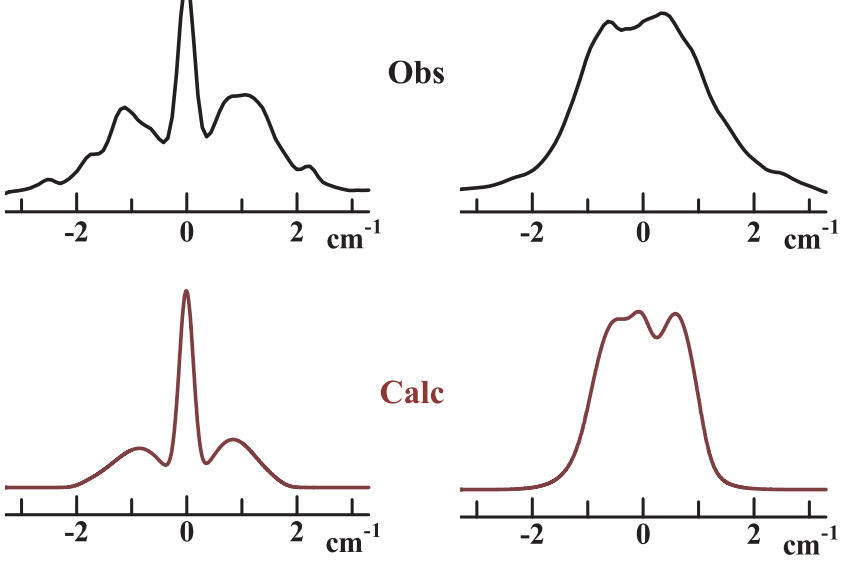

FIG. 6. Observed (top) and calculated (bottom) rotational contours of (a) $0_{0}^{0}$ band and (b) $61_{0}^{1}(e)$ band of sumanene. See text for the details of calculation.

mainly represented by $\Phi_{68}($ HOMO: $E) \rightarrow \Phi_{70}($ LUMO: $E)$ and $\Phi_{69}$ (HOMO: $E$ ) $\rightarrow \Phi_{71}$ (LUMO: $E$ ) excitations.

The $S_{1}{ }^{1} A_{1} \leftarrow S_{0}{ }^{1} A_{1}$ transition moment is along the oblate symmetric top axis $(c)$. We observed the rotational contour of the $0_{0}^{0}$ band, as shown in Fig. 6(a). A sharp $Q$ peak can clearly be seen at the band center, indicating that the transition moment is actually along the $c$ axis and perpendicular to the pivot phenyl ring, which is the benzene frame at the molecular center. This is considered to be the first evidence of an out-ofplane $\pi \pi^{\star}$ transition moment for aromatic hydrocarbons, and it has an important bowl effect on the electronic transition. The molecule is nonplanar. In this case, "out of plane" means perpendicular to the pivot phenyl ring. In contrast, the rotational contour of the $61_{0}^{1}$ band did not exhibit a $Q$ peak, as shown in Fig. 6(b). The transition moment for this $e$ vibronic band is, therefore, considered to be perpendicular to the $c$ axis.

We performed assignments referring to the vibrational energies in the $S_{1}$ state calculated by TDDFT(B3LYP)/6-31G, which were scaled by 0.977 . The results are listed in Table V and the assignments are shown with the expanded spectrum in Fig. 7. The observed prominent bands are assigned to $e$ vibrations, such as $v_{49}, v_{54}, v_{55}, v_{56}, v_{58}, v_{59}$, and $v_{61}$. Each fundamental band is followed by the combination bands with $a_{1}$ vibration of $v_{16}$ and $v_{17}$. The observed vibrational energies have good coincidence with the calculated values. We could identify the $31_{0}^{1}\left(a_{2}\right)$ combination band at $527 \mathrm{~cm}^{-1}$.

The dispersed fluorescence spectrum for the $61_{0}^{1}$ band excitation is shown in Fig. 8. The observed energies and the assignments referring to the results of the DFT(B3LYP)/6$31 \mathrm{G}(\mathrm{d}, \mathrm{p})$ calculation with a scaling factor of 0.981 are also listed in Table VI. We could identify the $61_{1}^{1}$ and $61_{2}^{1}$ bands and other $e$ vibrations of $v_{55}, v_{58}$, and $v_{59}$. These are followed by the combination bands of $a_{1}$ vibrations of $v_{4}, v_{13}, v_{14}, v_{16}$, and $v_{17}$.

\section{DISCUSSION}

We could observe the $0_{0}^{0}$ band of the $S_{1} \leftarrow S_{0}$ transition of jet-cooled sumanene, whose rotational contour showed a
TABLE V. Observed and calculated vibrational energies $\left(\mathrm{cm}^{-1}\right)$ and assignments in the $S_{1}{ }^{1} A_{1}$ states of sumanene.

\begin{tabular}{rcc}
\hline \hline Obs $^{\mathrm{a}}$ & Calc $^{\mathrm{b}}$ & Assignment \\
\hline 0 & 0 & $0_{0}^{0}\left(27943 \mathrm{~cm}^{-1}\right)$ \\
139 & 141 & $17_{0}^{1}\left(a_{1}\right)^{\mathrm{c}}$ \\
222 & 217 & $61_{0}^{1}(e)^{\mathrm{d}}$ \\
360 & 358 & $17_{0}^{1} 61_{0}^{1}$ \\
394 & 396 & $59_{0}^{1}(e)$ \\
414 & 422 & $58_{0}^{1}(e)$ \\
433 & 428 & $16_{0}^{1} 61_{0}^{1}$ \\
501 & 499 & $17_{0}^{2} 61_{0}^{1}$ \\
511 & 514 & $56_{0}^{1}(e)$ \\
527 & 517 & $31_{0}^{1} 61_{0}^{1}$ \\
532 & 537 & $17_{0}^{1} 59_{0}^{1}$ \\
548 & 549 & $55_{0}^{1}(e)$ \\
554 & 563 & $17_{0}^{1} 58_{0}^{1}$ \\
603 & 607 & $16_{0}^{1} 59_{0}^{1}$ \\
650 & 658 & $17_{0}^{1} 56_{0}^{1}$ \\
661 & 675 & $54_{0}^{1}(e)$ \\
667 & 655 & $17_{0}^{1} 31_{0}^{1} 61_{0}^{1}$ \\
688 & 690 & $17_{0}^{1} 55_{0}^{1}$ \\
758 & 760 & $16_{0}^{1} 55_{0}^{1}$ \\
795 & 785 & $14_{0}^{1} 61_{0}^{1}$ \\
800 & 816 & $17_{0}^{1} 54_{0}^{1}$ \\
\hline \hline
\end{tabular}

${ }^{\text {a }}$ Determined using the fluorescence excitation spectrum with an accuracy of $\pm 1 \mathrm{~cm}^{-1}$. ${ }^{\mathrm{b}}$ Results of TDFFT(B3LYP)/6-31G are scaled by 0.977 .

${ }^{\mathrm{c}}\left(a_{1}\right)$ means the $a_{1}$ fundamental band.

d $(e)$ means the $e$ fundamental band.

sharp $Q$ peak at the band center. This indicates that the $S_{1}$ state is ${ }^{1} A_{1}$ and the $S_{1} \leftarrow S_{0}$ transition moment is along the threefold symmetric top axis. The $0_{0}^{0}$ band is, however, weaker than vibronic bands, which gain intensity from vibronic interaction with the ${ }^{1} E$ electronic states. For a planar aromatic hydrocarbon, the out-of-plane transition moment arises from the $\sigma \pi^{\star}$ transition and is negligibly small. For a round buckybowl, the $\pi \pi^{\star}$ transition partly gives the out-of-plane moment, and the

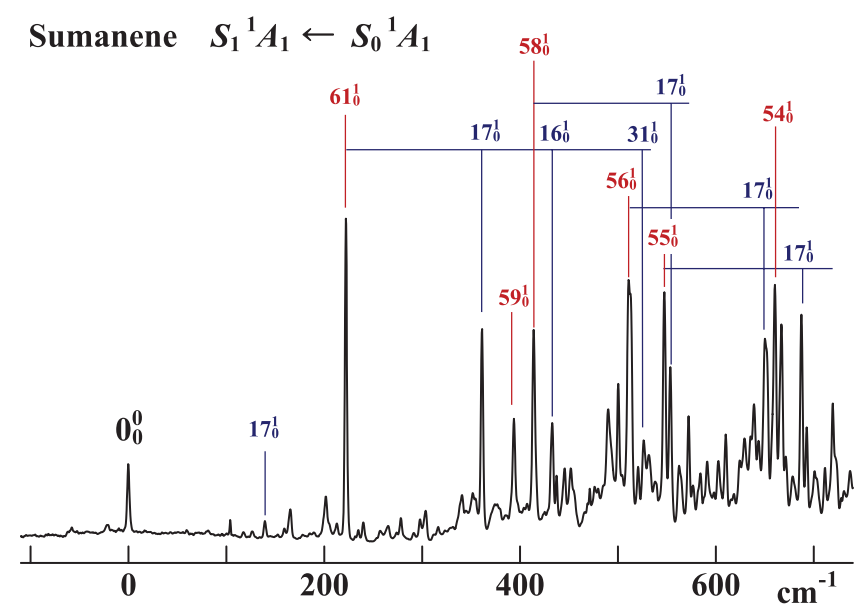

FIG. 7. Expanded fluorescence excitation spectrum of the $S_{1}{ }^{1} A_{1} \leftarrow S_{0}{ }^{1} A_{1}$ transition of jet-cooled sumanene and the vibronic assignments. 


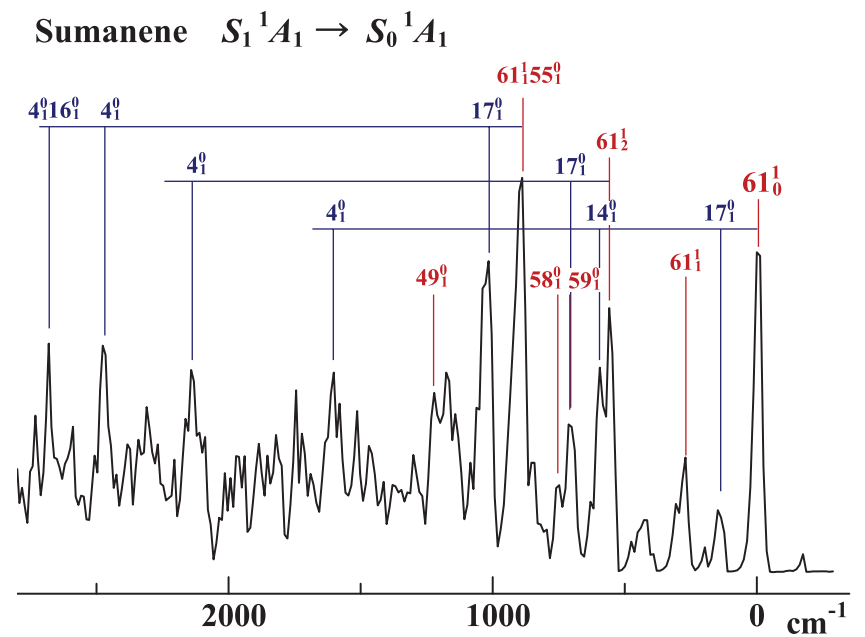

FIG. 8. Dispersed fluorescence spectra of jet-cooled sumanene for the $61_{0}^{1}$ band excitation.

${ }^{1} A_{1} \leftarrow S_{0}$ transition sumanene becomes allowed in $C_{3 v}$ symmetry. For a fullerene buckyball, the overall transition moment is cancelled out by the spherical symmetry, and only the residual part with structural distortion contributes to the electronic transition.

Next we discuss the vibrational modes, which are prominent in the observed spectra. The intensity of a vibronic band in the electronic spectrum depends on the Franck-Condon factor, which becomes large if the normal mode includes nuclear

TABLE VI. Observed and calculated vibrational energies $\left(\mathrm{cm}^{-1}\right)$ and assignments in the $S_{0}{ }^{1} A_{1}$ states of sumanene.

\begin{tabular}{rll}
\hline \hline Obs $^{\mathrm{a}}$ & Calc $^{\mathrm{b}}$ & \multicolumn{1}{c}{ Assignment } \\
\hline 0 & \multicolumn{1}{c}{0} & $61_{0}^{1}\left(28156 \mathrm{~cm}^{-1}\right)$ \\
148 & 137 & $17_{1}^{0} 61_{0}^{1}\left(a_{1}\right)^{\mathrm{c}}$ \\
197 & 213 & $16_{1}^{0} 61_{0}^{1}\left(a_{1}\right)$ \\
270 & 280 & $61_{1}^{1}(e)^{\mathrm{d}}$ \\
307 & 316 & $31_{1}^{0} 61_{0}^{1}\left(a_{2}\right)^{\mathrm{e}}$ \\
559 & 560 & $61_{2}^{1}$ \\
595 & 564 & $14_{1}^{0} 61_{0}^{1}\left(a_{1}\right)$ \\
630 & 618 & $13_{1}^{0} 61_{0}^{1}\left(a_{1}\right)$ \\
713 & 697 or 691 & $17_{1}^{0} 61_{2}^{1}$ or $59_{1}^{0} 61_{1}^{1}$ \\
749 & 709 & $58_{1}^{0} 61_{1}^{1}$ \\
888 & 886 & $55_{1}^{0} 61_{1}^{1}$ \\
1016 & 1023 or 1004 & $17_{1}^{0} 55_{1}^{0} 61_{1}^{1}$ or $54_{1}^{0} 61_{1}^{1}$ \\
1085 & 1099 & $16_{1}^{0} 55_{1}^{0} 61_{1}^{1}$ \\
1142 & 1123 & $14_{1}^{0} 61_{2}^{1}$ \\
1222 & 1209 & $49_{1}^{0} 61_{1}^{1}(e)$ \\
1602 & 1595 & $41_{0}^{0} 61_{0}^{1}\left(a_{1}\right)$ \\
2142 & 2155 & $4_{1}^{0} 61_{2}^{1}$ \\
2476 & 2481 & $4_{1}^{0} 55_{1}^{0} 61_{1}^{1}$ \\
2681 & 2694 & $4_{1}^{0} 55_{1}^{0} 61_{1}^{1} 16_{1}^{0}$ \\
\hline \hline
\end{tabular}

a Determined using the dispersed fluorescence spectrum with an accuracy of $\pm 12 \mathrm{~cm}^{-1}$. ${ }^{\mathrm{b}}$ Results of DFT(B3LYP)/6-31G(d,p) are scaled by 0.981 .

${ }^{\mathrm{c}}\left(a_{1}\right)$ means the $a_{1}$ fundamental band.

${ }^{\mathrm{d}}(e)$ means the $e$ fundamental band.

${ }^{\mathrm{e}}\left(a_{2}\right)$ means the $a_{2}$ fundamental band.
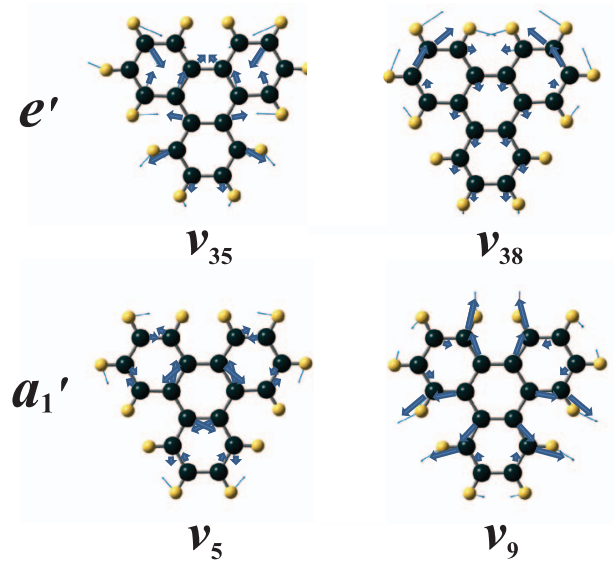

FIG. 9. Normal coordinates of $v_{35}\left(e^{\prime}\right), v_{38}\left(e^{\prime}\right), v_{5}\left(a_{1}^{\prime}\right)$, and $v_{9}\left(a_{1}^{\prime}\right)$ of triphenylene.

displacements of large structural changes upon the electronic excitation. This normal mode presumably plays an important role in the dynamical processes. The normal coordinates of vibration for the prominent bands in the spectra of triphenylene are illustrated in Fig. 9. A strong band appears if the equilibrium molecular structure is markedly different between the $S_{0}$ and $S_{1}$ states. In the $e^{\prime}$ vibration, the whole molecule deforms with homogeneous displacements of all nuclei, as seen in $v_{35}$ and $\nu_{38}$. The $a_{1}^{\prime}$ vibration can be seen as a combination band with the $e^{\prime}$ mode. In the $v_{5}\left(a_{1}^{\prime}\right)$ mode, the $\mathrm{C}-\mathrm{C}$ bond lengths in the pivot phenyl ring change alternately, which is similar to Kekulè vibration of the benzene molecule. In contrast, the $\mathrm{C}-\mathrm{C}$ bond lengths change uniformly in the $v_{9}\left(a_{1}^{\prime}\right)$ mode. This mode can be expressed as a breathing vibration of the pivot phenyl ring. It corresponds to the $v_{1}$ mode of benzene, whose vibronic bands are strongly observed in the $S_{1}{ }^{1} B_{2 u}$ $\leftarrow S_{0}{ }^{1} A_{1 g}$ transition. These Kekulè and breathing modes include large displacements of $\mathrm{C}$ nuclei. The $\pi$ bond orders and bond lengths are appreciably modified upon the $S_{1}{ }^{1} A_{1}^{\prime}$ $\leftarrow S_{0}{ }^{1} A_{1}^{\prime}$ electronic excitation of triphenylene as pointed out by Kokkin et al. ${ }^{15}$ The number of strong vibronic bands is small in triphenylene, indicating that, among a large number of normal modes, only these vibrational modes are vibronically active. This mode selectivity in nonadiabatic interaction is presumably due to its molecular planarity and high symmetry.

It should be noted that the vibronic bands of triphenylene$d_{12}$ are markedly weak in the high vibrational energy region above $1000 \mathrm{~cm}^{-1}$. We consider this behavior of triphenylene to be similar to that of benzene, which is called channel three. ${ }^{20-22}$ The fluorescence quantum yield becomes extremely small at an excess energy of $3200 \mathrm{~cm}^{-1}$ for benzene- $h_{6}$, and $2300 \mathrm{~cm}^{-1}$ for benzene- $d_{6}$. It has been shown by high-resolution spectroscopy that this is attributed to internal conversion (IC) assisted by intramolecular vibrational redistribution (IVR) due to the Coriolis interaction at high vibrational levels in the $S_{1}{ }^{1} B_{2 u}$ state. ${ }^{23,24}$ Intersystem crossing (ISC) to the triplet state is considered to be very slow for planar aromatic hydrocarbons. ${ }^{25,26}$ The mechanism of the deuterium effect in channel three has not yet been identified. Radiationless transitions are generally suppressed by deuterium 

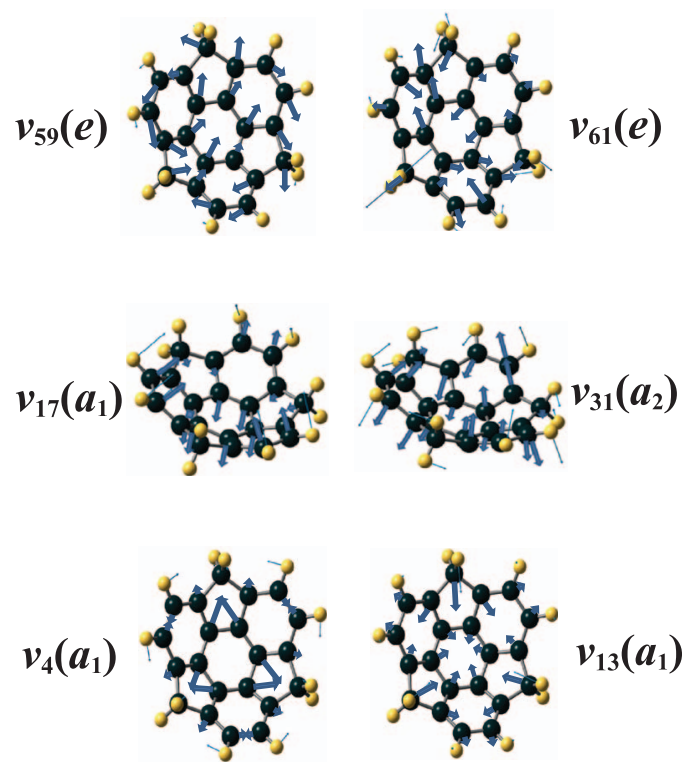

FIG. 10. Normal coordinates of $v_{59}(e), v_{61}(e), v_{17}\left(a_{1}\right), v_{31}\left(a_{2}\right), v_{4}\left(a_{1}\right)$, and $v_{13}\left(a_{1}\right)$ of sumanene.

substitution because of the shrinkage of the vibrational wave function. In fact, the fluorescence lifetime of triphenylene- $d_{12}$ (85 ns) at the $38^{1}$ level was longer than that of triphenylene$h_{12}(63 \mathrm{~ns})$. Our results indicate that triphenylene also possesses a similar channel, in which IC and IVR are enhanced in high vibrational levels of the $S_{1}$ state by deuterium substitution. The threshold energy is expected to be lower in triphenylene because the vibrational level density is much higher than in benzene.

Several normal coordinates of vibrations for the prominent bands in the spectra of sumanene are illustrated in Fig. 10. The $e$ vibrations such as $v_{59}$ and $v_{61}$ are deforming over the whole molecule, which include displacements of all nuclei in sumanene. We observed numerous $e$ vibronic bands and their combination bands with totally symmetric $a_{1}$ bands. The $v_{4}$ and $v_{13}$ modes correspond to the Kekulè and breathing modes in triphenylene, respectively. This indicates that the stable structures are appreciably different between the $S_{0}$ and $S_{1}$ states for the pivot phenyl ring, similar to triphenylene. The activity of these modes is reasonable because the electronic wavefunctions of sumanene are similar to those of triphenylene.

We also found the combination bands with $v_{17}\left(a_{1}\right)$ and $v_{31}\left(a_{2}\right)$ modes in the fluorescence excitation spectrum. These modes mostly consist of out-of-plane (perpendicular to the pivot phenyl ring) nuclear displacements, which change the curvature of its round structure. This is considered to be very important as a promoting mode for the bowl-to-bowl inversion. This reaction was found to occur on a time scale of $10 \mathrm{~h}$ in solution at room temperature. ${ }^{2,8,10}$ The barrier height to inversion was estimated to be approximately $20 \mathrm{kcal} / \mathrm{mol}$ (approximately $7000 \mathrm{~cm}^{-1}$ ) by theoretical calculations. ${ }^{27,28}$ Although the reaction coordinate is not identical to a normal coordinate, it may also be possible that in-plane modes such as Kekulè $v_{4}$ and breathing $v_{13}$ assist the bowl-to-bowl inversion in combination with out-of-plane modes. The pivot phenyl ring momentarily shrinks by the $v_{4}$ and $v_{13}$ vibrations and the molecular frame approaches the planar structure, leading to the lower barrier height to inversion. These vibrational energies are greatly changed by solvent and the actual barrier height is considered to be low enough for the inversion reaction at room temperature.

In our measurement of the fluorescence excitation spectrum, the excess energy in the $S_{1}{ }^{1} A_{1}$ state is, at most, $2200 \mathrm{~cm}^{-1}$, so the reaction probability is considered to be extremely small. It is, however, possible that the inversion efficiently occurs at the high vibrational levels in the $S_{0}$ state, which are populated after IC from the lower vibrational levels in the $S_{1}$ state. Although we could not see evidence of the decrease in fluorescence quantum yield as seen in triphenylene$d_{12}$, the main nonradiative process in the $S_{1}$ state of sumanene is expected to be IC to the hot $S_{0}$ state. This suggests that the bowl-to-bowl inversion can be enhanced by UV light irradiation. Although the fluorescence quantum yield in the $S_{1}{ }^{1} A_{1}$ state of sumanene could not be estimated by our experiments, it is considered to be comparable with the value of triphenylene (0.084). ${ }^{15}$

\section{CONCLUSIONS}

We summarize the difference in fluorescence excited spectra of jet-cooled triphenylene and sumanene as the bowl effects of the $\pi$ system. Both molecules possess a three-fold oblate symmetric top axis (principal axis $c$ ). The $S_{1} \leftarrow S_{0}$ transition is forbidden for the planar triphenylene molecule, but allowed for the nonplanar sumanene molecule. The transition moment is along the $c$ axis and perpendicular to the pivot phenyl ring (out of plane), which has been confirmed by the rotational contour of the $0_{0}^{0}$ band.

The spectrum of sumanene is much more congested than that of triphenylene. It is attributed to its lower symmetry and large structural change upon the electronic excitation. Some of the observed vibronic bands were assigned to normal modes which include the in-plane nuclear displacements of the pivot phenyl ring. These vibrations can lower the barrier height to bowl-to-bowl inversion by mixing with out-of-plane vibrational modes. The vibronic bands of sumanene are remarkably strong up to $2200 \mathrm{~cm}^{-1}$, indicating that radiationless transitions are not markedly enhanced by out-of-plane structural distortion.

\section{ACKNOWLEDGMENTS}

This work was partly supported by the Nanotechnology Platform Program (Molecule and Material Synthesis) of the Ministry of Education, Culture, Science and Technology (MEXT), Japan. The experiment was supported by the InterUniversity Network for Efficient Utilization of Chemical Research Equipments.

\footnotetext{
${ }^{1}$ S. Higashibayashi and H. Sakurai, Chem. Lett. 40, 122 (2011).

${ }^{2}$ T. Amaya and T. Hirao, Chem. Commun. 47, 10524 (2011).

${ }^{3}$ A. Sygula, Eur. J. Org. Chem. 2011, 1611.

${ }^{4}$ Y.-T. Wu and J. S. Siegel, Chem. Rev. 106, 4843 (2006).

${ }^{5}$ V. M. Tsefrikas and L. T. Scott, Chem. Rev. 106, 4868 (2006).
} 
${ }^{6}$ L. T. Scott, M. M. Hashemi, and M. S. Bratcher, J. Am. Chem. Soc. 114, 1920 (1992).

${ }^{7}$ T. J. Seiders, K. K. Baldridge, G. H. Grube, and J. S. Siegel, J. Am. Chem. Soc. 123, 517 (2001).

${ }^{8}$ T. Amaya and T. Hirao, Pure Appl. Chem. 84, 1089 (2012).

${ }^{9}$ S. Higashibayashi, R. Tsuruoka, Y. Soujanaya, U. Purushotham, G. N. Sastry, S. Seki, T. Ishikawa, S. Toyota, and H. Sakurai, Bull. Chem. Soc. Jpn. 85, 450 (2012).

${ }^{10}$ J. Mack, Y. Morita, S. Higashibayashi, H. Sakurai, and N. Kobayashi, Chem. Phys. Lett. 556, 188 (2013).

${ }^{11}$ H. Sakurai, T. Daiko, and T. Hirao, Science 301, 1878 (2003).

${ }^{12}$ W. E. Barth and R. G. Lawton, J. Am. Chem. Soc. 88, 380 (1966).

${ }^{13}$ H. Chojnacki, Z. Laskowski, A. Lewanowicz, Z. Ruziewicz, and R. Wandas, Chem. Phys. Lett. 124, 478 (1986).

${ }^{14}$ E. D. Donato, D. Vanzo, M. Semeraro, A. Credi, and F. Negri, J. Phys. Chem. A 113, 6504 (2009).

${ }^{15}$ D. L. Kokkin, N. J. Reilly, T. P. Troy, K. Nauta, and T. W. Schmidt, J. Chem. Phys. 126, 084304 (2007).

${ }^{16}$ M. J. Frisch, G. W. Trucks, H. B. Schlegel et al., GAUSSIAN 09, Revision C.01, Gaussian, Inc., Wallingford, CT, 2010.
${ }^{17}$ B. K. Selinger and W. R. Ware, J. Chem. Phys. 53, 3160 (1970).

${ }^{18}$ M. Baba, K. Mori, M. Yamawaki, K. Akita, M. Ito, S. Kasahara, and T. Yamanaka, J. Phys. Chem. A 110, 10000 (2006).

${ }^{19}$ M. Baba, M. Saitoh, Y. Kowaka, K. Taguma, K. Yoshida, Y. Semba, S. Kasahara, Y. Ohshima, T. Yamanaka, Y.-C. Hsu, and S. H. Lin, J. Chem. Phys. 131, 224318 (2009).

${ }^{20}$ J. H. Callomon, J. E. Parkin, and R. Lopez-Delgado, Chem. Phys. Lett. 13, 125 (1972).

${ }^{21}$ K. G. Spears and S. A. Rice, J. Chem. Phys. 55, 5561 (1971).

${ }^{22}$ A. S. Abramson, K. G. Spears, and S. A. Rice, J. Chem. Phys. 56, 2291 (1972).

${ }^{23}$ E. Riedle, Th. Weber, U. Schubert, H. J. Neusser, and E. W. Schlag, J. Chem. Phys. 93, 967 (1990), and the references therein.

${ }^{24}$ D. Y. Baek, J. H. Chen, J. G. Wang, A. Doi, S. Kasahara, M. Baba, and H. Katö, Bull. Chem. Soc. Jpn. 79, 75 (2006).

${ }^{25}$ M. A. El-Sayed, J. Chem. Phys. 38, 2834 (1963).

${ }^{26}$ M. Baba, J. Phys. Chem. A 115, 9514 (2011).

${ }^{27}$ U. D. Priyakumar and G. N. Sastry, J. Phys. Chem. A 105, 4488 (2001).

${ }^{28}$ T. C. Dinadayalane and G. N. Sastry, Tetrahedron 59, 8347 (2003). 